\title{
Assessment of Kidney Safety Parameters Among Hiv-Infected Patients Starting a Tenofovir-Containing Antiretroviral Therapy
}

\author{
Roberto Manfredi* and Leonardo Calza
}

Department of Internal Medicine, Ageing and Nephrologic Diseases, Division of Infectious Diseases, "Alma Mater Studiorum" University of Bologna, S. Orsola-Malpighi Hospital, Bologna, Italy

\begin{abstract}
To better evaluate the renal safety profile of tenofovir, we performed a retrospective study of HIV-infected naïve patients starting a first antiretroviral therapy between July 2005 and July 2008, and followed-up for 24 months. On the whole, 324 patients were enrolled: 201 tenofovir-exposed patients were compared with 123 tenofovir-unexposed subjects. In both unadjusted and adjusted analysis, tenofovir-exposed subjects had a significantly greater decline in glomerular filtration rate (GFR), and a significantly higher incidence of proximal tubular dysfunction through 24 months. Reduced glomerular and tubular functions were significantly associated with older age, diabetes, hypertension, and concomitant therapy with a HIV protease inhibitor.
\end{abstract}

Keywords: HIV, tenofovir, nephrotoxicity, glomerular filtration rate, tubulopathy.

\section{INTRODUCTION}

A growing number of case reports and cohort studies have described an association between treatment with tenofovir and renal tubulopathy or impaired glomerular filtration rate (GFR) in patients with human immunodeficiency virus (HIV) infection [1-3]. Alterations on tenofovir secretion by proximal renal tubule may lead directly to a greater drug accumulation in the renal tubular cells and, consequently, to a tubular damage and renal toxicity $[4,5]$.

We performed a retrospective cohort analysis of HIV-1infected adult patients naïve to antiretroviral therapy and initiating a first antiretroviral treatment in our Division of Infectious Diseases from July 1, 2005 through June 30, 2008.

\section{PATIENTS AND METHODS}

Patients starting a tenofovir-containing regimen were compared with those starting a tenofovir-sparing regimen and all enrolled subjects were followed-up for 24 months.

Patients were excluded if they had a prior diagnosis of renal disease or dialysis, any change in current antiretroviral regimen, or no follow-up clinical or laboratory data for at least 24 months.

We recorded following demographic, clinical, and laboratory data at the start of therapy and at 3-month intervals during the 24-month follow-up: sex, age, race, arterial pressure, clinical manifestations of renal disease, plasma HIV RNA, CD4 lymphocyte count, serum creatinine, serum uric acid, serum phosphate, serum potassium, evidence of proteinuria, and evidence of glucosuria in the presence of normal serum glucose $(<120 \mathrm{mg} / \mathrm{dL})$.

*Address correspondence to this author at the Infectious Diseases, University of Bologna, S. Orsola Hospital, Via Massarenti 11, I-40138 Bologna, Italy; Tel: +39051 6363355; Fax: +39 051343500 ;

E-mail: Roberto.manfredi@unibo.it
The glomerular filtration rate (GFR) was calculated using the four-variable Modification of Diet in Renal Disease (MDRD) formula [6], adjusted for body surface area, as usual.

Proximal tubular dysfunction was diagnosed with two or more of the following abnormalities: proteinuria (defined as $\geq 1+$ or $\geq 30 \mathrm{mg} \%$ on urine dipstick), glucosuria (defined as $\geq 1+$ or $\geq 30 \mathrm{mg} \%$ on urine dipstick), in the presence of normal serum glucose, hypouricemia $(<2 \mathrm{mg} / \mathrm{dL})$, hypophosphatemia $(<2.7 \mathrm{mg} / \mathrm{dL})$, and hypokalemia $(<3.5 \mathrm{mEq} / \mathrm{L})$.

We report multivariate hazard ratios (HR, Cox modeling) and linear outcomes (repeated measures) with predictors retained if $P<0.10$ (backward selection).

We analyzed continuous outcomes of changes in GFR, serum creatinine, and CD4 cell count through repeated measures linear mixed models. We employed Cox proportional hazard modeling for dichotomous outcomes of GFR $50 \%$ or higher decline, development of proximal tubular dysfunction, and achieving plasma HIV RNA $<50$ copies $/ \mathrm{mL}$.

Potential predictor variables included in multivariate models were age, gender, Black race, baseline creatinine, baseline CD4 lymphocyte count, antiretroviral regimen, diabetes mellitus, arterial hypertension, malignancies, acquired immunodeficiency syndrome (AIDS), chronic hepatitis B and $\mathrm{C}$, current use of diuretics or angiotensin converting enzyme inhibitors.

\section{RESULTS}

Three hundred twenty-four patients started a first antiretroviral treatment during the study period and met all inclusion and exclusion criteria. Among these 324 enrolled patients, $201(62 \%)$ initiated tenofovir-containing regimens and $123(38 \%)$ initiated tenofovir-sparing regimens.

Baseline demographic, epidemiological, and laboratory features were comparable in both groups, but the tenofovir- 
exposed group showed a greater prevalence of injection drug abuse and chronic hepatitis B (Table 1).

Similarly, there was no statistically significant difference between tenofovir-exposed and tenofovir-sparing groups in percentage of patients with HIV RNA $<50$ copies/mL $(77.5$ vs $73.8 \%$, respectively; $P=0.18$ ) and in change in CD4 lymphocyte count $(+168 v s+189$ cells $/ \mu$, respectively; $P=0.21)$ at the end of follow-up.

Unadjusted and multivariable adjusted modeling for renal-associated outcome parameters are summarized in Table 2. In both unadjusted and adjusted analysis for mean variation in GFR from baseline, the tenofovir-exposed subjects had statistically significant greater declines in GFR through 24 months compared with the tenofovir-sparing patients, and this decline was more significant among tenofovir-exposed persons with a higher baseline GFR $\left(>80 \mathrm{~mL} / \mathrm{min} / 1.73 \mathrm{~m}^{2}\right)$. The only statistically significant predictors $(P<0.05)$ of greater than $50 \%$ decline in GFR or as a continuous outcome measure were increased age ( $>50$ years), diabetes mellitus, arterial hypertension, chronic hepatitis $\mathrm{C}$, and inclusion of a protease inhibitor (PI) in the antiretroviral regimen. Increases in serum creatinine were also significantly greater among tenofovir-exposed patients compared with tenofovirunexposed ones, and statistically significant predictors $(P<0.05)$ of increased serum creatinine were higher age, diabetes, and lower CD4 count $(<200$ cells $/ \mu \mathrm{L})$.

A significantly higher percentage of tenofovir-exposed subjects did meet criteria for proximal tubular dysfunction at the end of 24-month follow-up compared with tenofovirsparing ones $(7.8 \%$ vs $1.3 \%$, respectively; $P<0.001)$. Particularly, the following components of the tubular damage definition showed a significantly greater incidence among tenofovir-exposed compared with tenofovir-unexposed patients: proteinuria $(19.5 \%$ vs $10.2 \%$, respectively; $P=0.003)$, glucosuria $(5.1 \%$ vs $0.7 \%$, respectively; $P<0.001)$, hypouricemia $(2.1 \%$ vs $0.4 \%$, respectively; $P=0.021)$, and hypophosphatemia (7.8\% vs $1.4 \%$, respectively; $P=0.009)$.

Statistically significant predictors $(P<0.05)$ of development of proximal tubular dysfunction were higher age, diabetes mellitus, arterial hypertension, and a PI-based regimen.

Table 1. Baseline Demographic, Epidemiological, and Laboratory Features of the 324 Patients Included in our Study

\begin{tabular}{|c|c|c|c|}
\hline Parameters & Tenofovir-exposed patients $(\mathrm{n}=\mathbf{2 0 1})$ & Tenofovir-unexposed patients $(\mathrm{n}=123)$ & Pvalue \\
\hline Male, n (\%) & $145(72.1)$ & $92(74.8)$ & 0.82 \\
\hline Black, n (\%) & $11(5.4)$ & $5(4.1)$ & 0.71 \\
\hline IDU, n (\%) & $28(13.9)$ & $7(5.7)$ & 0.03 \\
\hline H/BSX, n (\%) & $108(53.7)$ & $74(60.1)$ & 0.58 \\
\hline Age, years ${ }^{1}$ & $42.5(34.2-50.1)$ & $42.9(33.6-51.4)$ & 0.48 \\
\hline Diabetes mellitus, n (\%) & $12(5.9)$ & $7(5.7)$ & 0.91 \\
\hline Arterial hypertension, $\mathrm{n}(\%)$ & $25(12.4)$ & $14(11.4)$ & 0.67 \\
\hline Malignancies, n (\%) & $15(7.5)$ & $9(7.3)$ & 0.79 \\
\hline Chronic hepatitis B, n (\%) & $19(9.4)$ & $6(4.9)$ & 0.006 \\
\hline Chronic hepatitis C, n (\%) & $28(13.9)$ & $13(10.6)$ & 0.56 \\
\hline AIDS, n (\%) & $29(14.4)$ & $16(13)$ & 0.66 \\
\hline CD4 lymphocyte count $(\text { cells } / \mu \mathrm{L})^{1}$ & $215(88-301)$ & $207(91-289)$ & 0.44 \\
\hline HIV RNA $\left(\log _{10} / \mathrm{mL}\right)^{1}$ & $4.4(3.9-5.1)$ & $4.6(3.9-5.2)$ & 0.81 \\
\hline Creatinine $(\mathrm{mg} / \mathrm{dL})^{1}$ & $0.87(0.71-1.05)$ & $0.82(0.73-1.02)$ & 0.72 \\
\hline $\operatorname{GFR}\left(\mathrm{mL} / \mathrm{min} / 1.73 \mathrm{~m}^{2}\right)^{1}$ & $96.2(83.2-111.4)$ & $93.1(82.1-109.4)$ & 0.75 \\
\hline Current use of diuretics, $n(\%)$ & $17(8.5)$ & $9(7.3)$ & 0.71 \\
\hline Current use of ACE inhibitors, n (\%) & $21(10.4)$ & $11(8.9)$ & 0.77 \\
\hline $\begin{array}{ll}\text { Current antiretroviral agents, } \mathrm{n}(\%) \\
- & \text { abacavir } \\
- & \text { zidovudine } \\
- & \text { stavudine } \\
- & \text { lamivudine } \\
- & \text { emtricitabine } \\
- & \text { lopinavir/ritonavir } \\
- & \text { atazanavir/ritonavir } \\
- & \text { fosamprenavir/ritonavir } \\
- & \text { nevirapine } \\
- & \text { efavirenz }\end{array}$ & $\begin{array}{c}0 \\
0 \\
0 \\
45(22.4) \\
156(77.6) \\
25(12.4) \\
56(27.9) \\
5(2.5) \\
17(8.4) \\
98(48.7)\end{array}$ & $\begin{array}{c}78(63.4) \\
25(20.3) \\
2(1.6) \\
119(96.7) \\
4(3.2) \\
11(8.9) \\
34(27.6) \\
1(0.8) \\
11(8.9) \\
66(53.6)\end{array}$ & $\begin{array}{c}<0.001 \\
<0.001 \\
0.65 \\
<0.001 \\
<0.001 \\
0.45 \\
0.92 \\
0.52 \\
0.97 \\
0.49\end{array}$ \\
\hline
\end{tabular}

IDU, injective drug users; H/BSX, homosexuals/bisexuals; AIDS, acquired immunodeficiency syndrome; GFR, estimated glomerular filtration rate by MDRD formula, adjusted per body surface area; ACE, angiotensin converting enzyme.

${ }^{1}$ Median value (interquartile range). 
Table 2. Unadjusted and Multivariable Adjusted Modeling for Parameters Associated with Renal Function, in the Studied Patient Population

\begin{tabular}{|c|c|c|c|c|}
\hline \multirow{2}{*}{ Parameters } & \multicolumn{4}{|c|}{ Values obtained when comparing Tenofovir-exposed with Tenofovir-unexposed ${ }^{1}$ subjects $^{-}$} \\
\hline & Unadjusted & Pvalue & Adjusted model $^{2}$ & Pvalue \\
\hline $\begin{array}{c}\text { Change in } \mathrm{GFR}^{3} \text { at } 24 \text { months: } \\
\text { - overall } \\
-50<\text { baseline GFR } \leq 80 \\
\text { - baseline GFR }>80\end{array}$ & $\begin{array}{l}-5.9(-8.1 \text { to }-3.7) \\
-2.8(-4.7 \text { to }-0.9) \\
-6.4(-8.6 \text { to }-4.3)\end{array}$ & $\begin{array}{c}<0.001 \\
0.003 \\
<0.001\end{array}$ & $\begin{array}{l}-5.1(-7.6 \text { to }-3.8) \\
-2.1(-4.2 \text { to }-0.6) \\
-5.8(-8.1 \text { to }-3.9)\end{array}$ & $\begin{array}{c}<0.001 \\
0.005 \\
<0.001\end{array}$ \\
\hline GFR decreased $>50 \%$ from baseline hazard ratio & $1.62(0.91$ to 2.65$)$ & 0.065 & $1.45(0.82$ to 2.31$)$ & 0.078 \\
\hline Change in $\mathrm{SCr}^{4}$ & $+0.09(+0.06$ to +0.11$)$ & 0.021 & $+0.06(+0.04$ to +0.09$)$ & 0.035 \\
\hline Development proximal tubular dysfunction hazard ratio & $6.12(2.75$ to 14.21$)$ & $<0.001$ & $5.78(2.34$ to 13.66$)$ & $<0.001$ \\
\hline
\end{tabular}

${ }^{1}$ Results are mean estimate (95\% confidence interval).

${ }^{2}$ Multivariate modeling by linear repeated measures for continuous outcomes and Cox proportional hazard modeling for dichotomous parameters. Predictor variables include age, gender, Black race, baseline creatinine, CD4 lymphocyte count, antiretroviral regimen, diabetes mellitus, arterial hypertension, malignancies, full-blown acquired immunodeficiency syndrome, chronic hepatitis B or C, current use of diuretics or angiotensin converting enzyme inhibitors. Final model developed by backward selection until all covariates were at $P$ $<0.10$ level.

${ }^{3}$ Estimated glomerular filtration rate (GFR) calculated by MDRD formula, adjusted per body surface area $\left(\mathrm{mL} / \mathrm{min} / 1.73 \mathrm{~m}^{2}\right)$

${ }^{4} \mathrm{SCr}$, serum creatinine $(\mathrm{mg} / \mathrm{dL})$.

Numbers of treatment discontinuations were comparable between tenofovir-exposed and tenofovir-sparing patients (18.2\% vs $16.5 \%$, respectively; $\mathrm{P}=0.53$ ).

\section{DISCUSSION}

Some observational studies have found evidence of a mild decrease in GFR in tenofovir-treated patients [7-10], but renal toxicity associated with this nucleotide analogue mostly occurs as tubular dysfunction, including Fanconi's syndrome and acute tubular necrosis $[2,3]$.

Exposure to tenofovir was found to be associated with an increased risk of tubular abnormalities, as showed by the evaluation of plasma and urine markers of tubulopathy.

The incidence of glucosuria, proteinuria, hyperphosphaturia, hyperaminoaciduria, and an increased excretion of retinol-binding protein and beta- 2 microglobulin, were significantly higher in tenofovir-treated subjects than in tenofovir-unexposed ones, and older age, PI-exposure, and comorbidities (mostly diabetes mellitus), acted as frequent clinical predictors of tenofovir-induced tubulopathy $[7,11$, 12].

On the other hand, large observational studies and clinical trials have shown no evidence of glomerular or tubular damage when tenofovir is employed as a component of the initial antiretroviral regimen [13-15].

Moreover, it is well known that all conditions of acute and chronic kidney failure are frequently associated with an increased risk in drug-related renal toxicities. This last condition is expected to become more frequent and more relevant just in the population of patients living with HIV, which will be exposed to multiple pharmacotherapy in the next years [16], whilst the mean age of HIV-infected individuals is always on increase [17], and a premature ageing is typical of HIV-infected subjects themselves [18].

In our retrospective study, tenofovir exposure was found to be associated with a significant decline in GFR and mostly with a significantly higher risk of tubular damage, in line with previously reported observational studies. Decline in glomerular function was more evident in patients with base- line higher GFR, and significant predictors of glomerular or tubular damage were represented by a proportionally older age, underlying comorbidities (especially diabetes mellltus and hypertension), and HIV PI use.

\section{CONCLUSION}

In conclusion, tenofovir seems associated with a potential negative impact on kidney glomerule and proximal tubule function and structure in HIV-infected patients, but longterm consequences of these renal abnormalities are still unknown, and a periodic monitoring of renal function parameters in all patients taking tenofovir as a part of an antiretroviral therapy is strongly adviced.

\section{REFERENCES}

[1] Barrios A, Garcia-Benayas T, Gozalez-Lahoz J, Soriano V. Tenofovir-related nephrotoxicity in HIV-infected patients. AIDS 2004; 18: 960-3.

[2] Nelson M, Katlama C, Montaner J, et al. The safety of tenofovir disoproxil fumarate for the treatment of HIV infection in adults: the first 4 years. AIDS 2007; 21: 1273-81.

[3] Izzedine H, Harris M, Perazella MA. The nephrotoxic effects of HAART. Nat Rev Nephrol 2009; 5: 563-73.

[4] Rodriguez-Novoa S, Labarga P, Soriano V, et al. Predictors of kidney tubular dysfunction in HIV-infected patients treated with tenofovir: a pharmacogenetic study. Clin Infect Dis 2009; 48: E108-16.

[5] Rodriguez-Novoa S, Labarga P, D'Avolio A, et al. Impairment in kidney tubular function in patients receiving tenofovir is associated with higher tenofovir plasma concentrations. AIDS 2010; 24: 10646.

[6] Levey AS, Coresh J, Greene T, et al. Using standardized serum creatinine values in the modification of diet in renal disease study equation for estimating glomerular filtration rate. Ann Intern Med 2006; 145: 247-54.

[7] Horberg M, Tang B, Towner W, et al. Impact of tenofovir on renal function in HIV-infected, antiretroviral-naïve patients. J Acquir Immune Defic Syndr 2010; 53: 62-9.

[8] Fux CA, Simcock M, Wolbers M, et al. Tenofovir use is associated with a reduction in calculated glomerular filtration rates in the Swiss HIV Cohort Study. Antivir Ther 2007; 12: 1165-73.

[9] Young B, Buchacz K, Baker RK, et al. Renal function in tenofovirexposed and tenofovir-unexposed patients receiving highly active antiretroviral therapy in the HIV Outpatient Study. J Int Assoc Physicians AIDS Care (Chic III) 2007; 6: 178-87.

[10] Overton ET, Nurutdinova D, Freeman J, Seyfried W, Mondy KE. Factors associated with renal dysfunction within an urban HIV- 
infected cohort in the era of highly active antiretroviral therapy. HIV Med 2009; 10: 343-50.

[11] Labarga P, Barreiro P, Martin-Carbonero L, et al. Kidney tubular abnormalities in the absence of impaired glomerular function in HIV patients treated with tenofovir. AIDS 2009; 23: 689-96.

[12] Post FA, Moyle GJ, Stellbrink HJ, et al. Randomized comparison of renal effects, efficacy, and safety with once-daily abacavir/lamivudine $v s$. tenofovir/emtricitabine, administered with efavirenz, in antiretroviral-naïve, HIV-1-infected adults: 48-week results from the ASSERT Study. J Acquir Immune Defic Syndr 2010; 55: 49-57.

[13] Gallant JE, Parish MA, Keruly JC, Moore RD. Changes in renal function associated with tenofovir disoproxil fumarate treatment, compared with nucleoside reverse-transcriptase inhibitor treatment. Clin Infect Dis 2005; 40: 1194-8.
[14] Arribas JR, Pozniak AL, Gallant JE, et al. Tenofovir disoproxil fumarate, emtricitabine, and efavirenz compared with zidovudine/lamivudine and efavirenz in treatment-naïve patients: 144-week analysis. J Acquir Immune Defic Syndr 2008; 47: 74-8.

[15] Smith KY, Patel P, Fine D, et al. Randomized, double-blind, placebo-matched, multicenter trial of abacavir/lamivudine or tenofovir/emtricitabine with lopinavir/ritonavir for initial HIV treatment. AIDS 2009; 23: 1547-56.

[16] Gruber VA, McCance-Katz EF. Methadone, buprenorphine, and street drug interactions with antiretroviral medications. Curr HIV/AIDS Rep 2010; 7: 152-60.

[17] Manfredi R. HIV infection and advanced age: emerging epidemiological, clinical, and management issues. Ageing Res Rev 2004; 3 : 31-54.

[18] Martin J, Volberding P. HIV and premature aging: a field still in its infancy. Ann Intern Med 2010; 153: 477-9.

(C) Manfredi and Calza; Licensee Bentham Open.

This is an open access article licensed under the terms of the Creative Commons Attribution Non-Commercial License (http://creativecommons.org/licenses/ by-nc/3.0/) which permits unrestricted, non-commercial use, distribution and reproduction in any medium, provided the work is properly cited. 